\title{
Mesenchymal Stem Cells Promote Cardiac Muscle Repair via Enhanced Neovascularization
}

\author{
Jianfeng Zhang ${ }^{\mathrm{a}, \mathrm{b}} \quad$ Yicheng $\mathrm{Wu}^{\mathrm{a}}$ Anqing Chen ${ }^{\mathrm{a}}$ Qiang Zhao \\ aDepartment of Cardiac Surgery, Ruijin Hospital, School of Medicine, Shanghai Jiao Tong University, \\ Shanghai, China, bDepartment of Cardiovascular Surgery, Shanghai Chest Hospital, Shanghai Jiao Tong \\ University, Shanghai, China
}

\section{Key Words}

Mesenchymal stem cells $\bullet$ Cardiac muscle repair $\bullet$ Vascular endothelial growth factor $\bullet$ Placental growth factor

\begin{abstract}
Background/Aims: Transplantation of bone-marrow derived stem/progenitor cells has an established therapeutic effect on cardiac muscle repair after injury. However, the exact mechanism that underlies this phenomenon is not completely understood. Methods: Here we transplanted mesenchymal stem cells (MSCs), a major population from the bone-marrow derived stem/progenitor cells, and studied its effects on cardiac muscle repair after injury. Results: MSCs transplantation significantly improved cardiac muscle repair after injury. The grafted MSCs did not significantly differentiate into cardiac muscle cells themselves, but appeared to induce neovascularization in the injured heart. In a loss-of-function experiment, we further show that production and secretion of placental growth factor, but not vascular endothelial growth factor A in MSCs, were essential for the MSCs-induced neovasularization after cardiac muscle injury to facilitate cardiac muscle repair. Conclusion: Our study thus sheds light on an undescribed role of placental growth factor in cardiac muscle regeneration.
\end{abstract}




\section{Introduction}

Bone marrow-derived stem/progenitor cells can mediate neovascularization in the ischemic myocardium and improve heart function $[1,2]$. However, the underlying mechanism remains unclear [3-5]. Moreover, significant variability of the responses to using bone marrow-derived stem/progenitor cells may largely result from the feature of bone marrow-derived stem/progenitor cells as a mixture of different cell types [3-6]. Mesenchymal stem cells (MSCs) are the most important population of bone marrow-derived stem/progenitor cells. Cells similar to bone marrow-derived MSCs have now been identified in all postnatal tissues, and play essential roles in the renewal and repair of various tissues [7-12]. Determination of the biological properties of MSCs has enabled successful revision of the role of MSCs in various organs and tissues [7-12].

In the current study, we addressed the question of whether MSCs are the functional part of bone marrow-derived stem/progenitor cells that is capable of promoting cardiac muscle repair after injury, and of what the underlying molecular mechanism is. We found that MSCs transplantation significantly improved cardiac muscle repair after injury. The grafted MSCs did not significantly differentiate into cardiac muscle cells themselves, but appeared to contribute to neovascularization in the injured heart, by production and secretion of placental growth factor (PLGF), but not vascular endothelial growth factor A (VEGF-A).

\section{Materials and Methods}

Mouse handling

All mouse experiments were approved by the Institutional Animal Care and Use Committee at Ruijin Hospital, Shanghai Jiaotong University School of Medicine (Animal Welfare Assurance). Surgeries were performed under ketamine/xylazine anesthesia, in accordance with the Principles of Laboratory Care, supervised by a qualified veterinarian. All efforts were made to minimize pain and suffering. Male C57BL/6 mice of 12 weeks of age were used in the current study. Six mice were analyzed in each experimental condition.

\section{Acute myocardial infarction (AMI) model}

A standardized mouse AMI model was applied as has been described before [13]. Briefly, after the mice were anesthetized with ketamine/xylazine, they were orally intubated with 22 GIV catheter and artificially ventilated with a respirator (Harvard Apparatus, Boston, MA, USA). A small oblique thoracotomy was performed lateral to the left intercostal line in the third costal space to expose the heart. After the pericardium was opened, the proximal left anterior descending artery (LAD) branch of the left coronary artery was ligated using 8-0 gauge polypropylene sutures through a dissecting microscope.

\section{MSCs transplantation}

For MSCs transplantation, $10^{6}$ MSCs were intravenously injected via tail vein to the mice immediately after surgical induction of AMI.

\section{Heart function evaluation}

Mice underwent echocardiography just before MI ( 0 week) and at 1, 2, and 4 weeks after AMI as has been described before [13]. Transthoracic echocardiography was performed with a 6- to 15-MHz transducer (SONOS 5500, Hewlett Packard, Los Angeles, CA, USA). Two-dimensional images were obtained in the parasternal long and short axis and apical 4-chamber views. M-mode images of the left ventricular (LV) short axis were taken just below the level of mid-papillary muscles. LV end-diastolic and end-systolic dimensions were measured and functional shortening was determined according to modified recommendations of the American Society of Echocardiography. A mean value of 3 measurements was determined for each time point.

MSCs isolation, culturing and differentiation

The MSCs were isolated and grown in culture as has been described previously $[11,14,15]$. Briefly, plugs of marrow from five 12-week-old male C57/6BL mice (about 30g body weight) were dispersed 
in Dulbecco's Modification of Eagle's Medium (DMEM, Life technologies, San Jose, CA, USA) and then centrifuged at $900 \mathrm{~g}$ for $5 \mathrm{~min}$. The pellets were re-suspended and plated at $10^{5} \mathrm{cells} / \mathrm{cm}^{2}$ in DMEM containing $10 \%$ FBS. After 10 passages' selection of attached cells, the cells were sorted for Stro-1 (Becton-Dickinson Biosciences, San Jose, CA, USA) by flow cytometry to get rid of contaminating cells. A positive clone was selected after subjection to chondrogenetic, osteogenic, and adipogenic differentiation assays to confirm phenotype. For chondrogenetic induction, $2.5 \times 10^{5} \mathrm{MSC}$ were induced with $5 \mathrm{ml}$ chondrogenetic induction medium containing $10 \mu \mathrm{g}$ transforming growth factor $\beta 1$ (R\&D System, Los Angeles, CA,USA), 50 $\mu$ g insulin growth factor 1 (R\&D System), and $2 \mathrm{mg} / \mathrm{mL}$ dexamethasone (DMSO, Sigma-Aldrich, St. Louis, MO, USA) followed by centrifugation at $500 \mathrm{~g}$ for $5 \mathrm{~min}$. The cell pellets were maintained in the chondrogenetic induction medium for 14 days and subjected to Alcian blue staining. For osteogenic induction, cells were digested and seeded onto a 24-well plate at a density of $10^{4} \mathrm{cells} /$ well, and then maintained in osteogenic induction medium containing 10nM Vitamin D3 (Sigma-Aldrich) and 10mM $\beta$-phosphoglycerol and $0.1 \mu \mathrm{M}$ DMSO for 14 days and were subjected to Von kossa staining. For adipogenic induction, cells were digested and seeded onto a 24-well plate at a density of $10^{4} \mathrm{cells} /$ well, and then maintained in the adipogenic induction medium containing $0.5 \mathrm{mM}$ 3-isobutyl-1-methylxanthine (IBMX), $200 \mu \mathrm{M}$ indomethacin, $10 \mu \mathrm{M}$ insulin and $1 \mu \mathrm{M}$ DMSO for 14 days and subjected to Oil red 0 staining.

\section{CFSE labeling of MSCS}

CFDA-SE (CFSE, Invitrogen, St. Louis, MO, USA) was prepared according to the manufacturer's instruction. Cultured cells were incubated with $10 \mu \mathrm{M}$ CFSE for 10 minutes, resulting in efficient sustained labeling of the cells, as has been described before $[16,17]$. The cells were then extensively washed with PBS to remove extracellular CFSE in the media before transplantation. CFSE-labeled MSCs were then purified by flow cytometry based on green fluorescence.

\section{Knockdown of VEGF-A or PLGF in MSCs}

Recombinant lenti-viruses expressing short hair interfering RNA (shRNAs) for VEGF-A or PLGF together with a GFP reporter under the control of CMV promoter (Invivogen Therapeutics, Toulouse, France) efficiently infected MSCs at MOI 100 and resulted in nearly 100\% infection efficiency, based on GFP expression. The sequence for shVEGF-A is 5'-TGTGAATGCAGACCAAAGA-3', as has been described before [18]. ShPLGF was purchased from Qiagen (Hilden, Germany).

\section{Flow cytometry}

The mouse hearts were digested into single cells as has been described before [19]. The digests were incubated either with M-cadherin antibody (M-Cad, Millipore, Billerica, MA, USA), or with CD31 antibody (Becton-Dickinson Biosciences), or F4/80 (ebioscience, San Diego, CA, USA) followed by labeling with PE-conjugated anti-mouse second antibody (Becton-Dickinson Biosciences). Afterwards, flow cytometry analysis was performed in a FACSAria flow cytometer. MSCs were recognized by direct fluorescence of CFSE.

\section{Immunohistochemistry}

Mouse hearts were dissected out and fixed with 4\% paraformaldehyde for 6 hours, and then cryoprotected in $20 \%$ sucrose overnight. Samples were then sectioned at $6 \mu \mathrm{M}$. For immunostaining, primary antibodies are rat anti-CD31 (Becton-Dickinson Biosciences) and rabbit anti-M-cadherin (M-Cad, Millipore). Secondary antibodies were Cy3- and Cy2- conjugated antibodies for corresponding species (Jackson Labs, Bar Harbor, ME, USA). CFSE was visualized by direct green fluorescence. DAPI was used to stain nucleus. Vessel density was determined with NIH ImageJ software by measuring the percentage of CD31-positive area to the total heart area. For quantification of heart fibrosis, heart sections were stained with Masson trichrome as has been described before [20]. Evaluation of heart fibrosis in each sample was performed based on 20 randomly selected fields per section, which were examined under $\times 400$ magnification for assessment of the degree of heart fibrosis by visualizing blue-stained areas, exclusive of staining that colocalized with perivascular or intramural vascular structures, the endocardium, or LV trabeculae. ImageJ software was used to determine blue-stained areas and non-stained myocyte areas from each section using a color-based threshold. The percentage of total fibrosis area was calculated as the summed blue-stained areas divided by total ventricular area, as has been described previously [21].

\section{KARGER}


Quantitative real-time PCR (RT-qPCR)

RNA was extracted from cultured cells with RNeasy (Qiagen) for cDNA synthesis. RT-qPCR was performed in duplicates with QuantiTect SYBR Green PCR Kit (Qiagen). All primers were purchased from Qiagen. Values of genes were normalized against $\alpha$-tubulin and then compared to the control $(=1)$.

ELISA

The protein levels of VEGF-A and PLGF were determined by a VEGF-A and a PLGF ELISA kit (Raybio, Norcross, GA, USA), respectively. ELISAs were performed according to the instructions of the manufacturer. Briefly, the cell lysates were added to a well coated with VEGF-A/PLGF polyclonal antibody, and then immunosorbented by biotinylated monoclonal anti-human VEGF-A/PLGF antibody at room temperature for 2 hours. The color development catalyzed by horseradish peroxidase was terminated with $2.5 \mathrm{~mol} / \mathrm{l}$ sulfuric acid and the absorption was measured at $450 \mathrm{~nm}$. The protein concentration was determined by comparing the relative absorbance of the samples with the standards. Each sample was assayed in duplicates and the mean value was taken for statistical analysis.

\section{Western blot}

Protein was extracted from the cultured cells by RIPA buffer (Sigma-Aldrich) for Western Blot. The supernatants were collected after centrifugation at $12000 \times \mathrm{g}$ at $4^{\circ} \mathrm{C}$ for $20 \mathrm{~min}$. Protein concentration was determined using BCA protein assay, and whole lysates were mixed with $4 \times$ SDS loading buffer $(125 \mathrm{mmol} / \mathrm{L}$ Tris-HCl, 4\% SDS, 20\% glycerol, $100 \mathrm{mmol} / \mathrm{L} \mathrm{DTT}$, and $0.2 \%$ bromophenol blue) at a ratio of 1:3. Samples were heated at $100{ }^{\circ} \mathrm{C}$ for $5 \mathrm{~min}$ and were separated on SDS-polyacrylamide gels. The separated proteins were then transferred to a PVDF membrane. The membrane blots were first probed with a primary antibody. After incubation with horseradish peroxidase-conjugated second antibody, autoradiograms were prepared using the enhanced chemiluminescent system to visualize the protein antigen. The signals were recorded using X-ray film. Primary antibodies for Western Blot are anti-phosphorylated-Flt-1 (pFlt-1, tyr1213, Millipore), anti-phosphorylated-ERK1/2 (pERK1/2, tyr202/204, Cell Signaling, San Jose, CA, USA), antiphosphorylated-Akt (pAkt, ser473, Cell Signaling) and anti- $\alpha$-tubulin (Cell Signaling). Secondary antibody is HRP-conjugated anti-rabbit (Jackson Labs). Images shown in the figure were representative from 5 repeats. Recombinant PLGF was purchased from Sigma-Aldrich, and used in a dose of $10 \mathrm{nmol} / \mathrm{l}$.

Statistics

All values are depicted as mean \pm standard deviation from 6 individuals and are considered significant if $\mathrm{p}<0.05$. All data were statistically analyzed using one-way ANOVA with a Bonferoni correction.

\section{Results}

Isolation, culturing, differentiation and labeling of primary mouse MSCs

Primary mouse MSCs were isolated from male C57BL/6 mice and expanded in culture. After 10 passages' selection of attached cells, the cells were sorted for Stro-1, the best MSC marker [22, 23], by flow cytometry to get rid of contaminating cells (Fig. 1A). Our data show that more than 95\% cultured cells are Stro-1-positive. Differentiation assays were performed, including Von kossa staining to evaluate osteogenic induction, Oil red O staining to evaluate adipogenic induction and Alcian blue staining to evaluate chondrogenetic, which confirmed MSCs phenotype (Fig. 1B-D). Immediately before transplantation, the MSCs were labeled with CFSE, to allow in vivo lineage tracing (Fig. 1E). Successfully labeled MSCs were purified by flow cytometry (Fig. 1F).

Grafted MSCs improved heart function after cardiac muscle injury in isogenic mice

In order to evaluate the effect of transplantation of MSCs on injured heart, we transplanted CFSE-labeled MSCs immediately after induction of AMI, as has been described before [13]. $\mathrm{LV}$ end-diastolic and end-systolic dimensions were measured and functional shortening was determined before AMI (week 0 ) and on 1, 2, 3 and 4 weeks post-AMI. The echocardiography 2, 3, 4 weeks post-AMI showed significantly improved LV diastolic dimensions (LVDd) (Fig. 
A

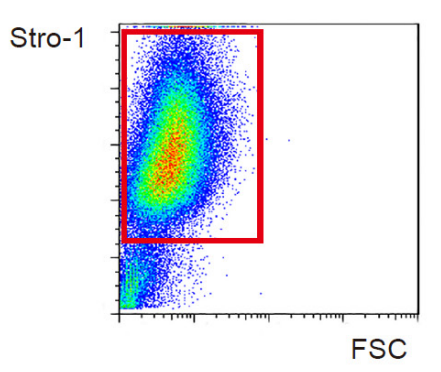

C

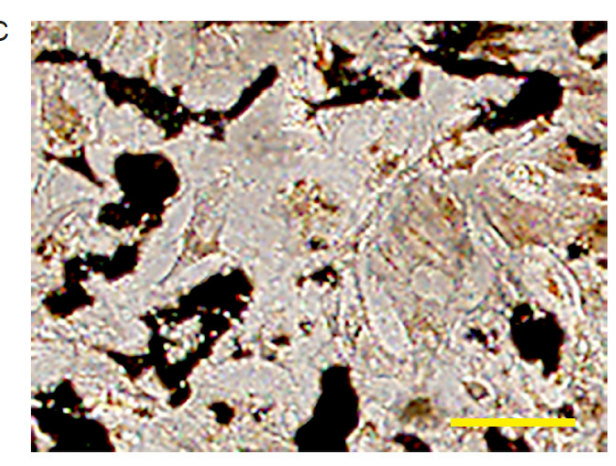

E

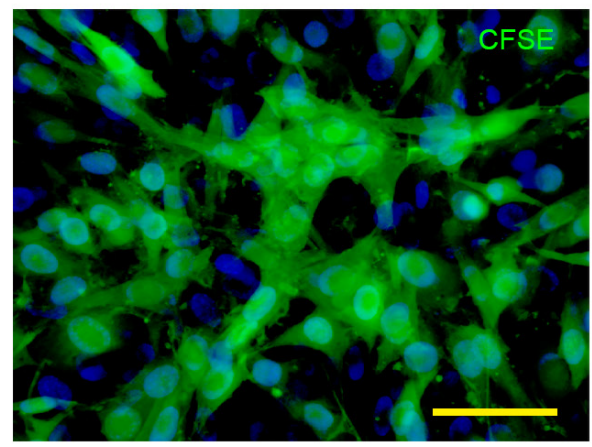

B

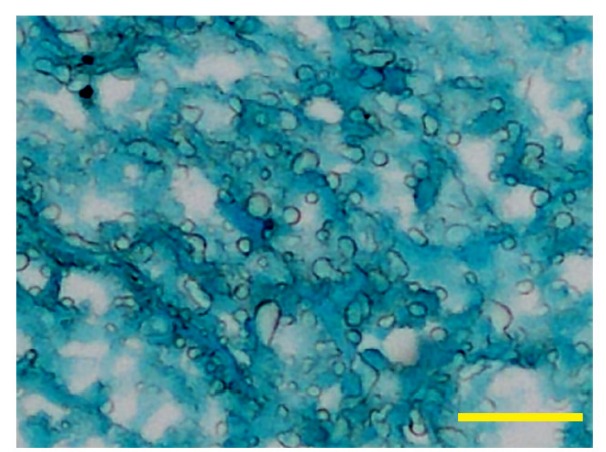

D

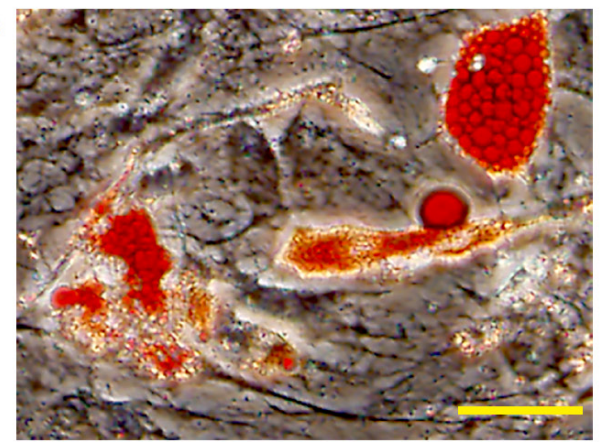

$\mathrm{F}$

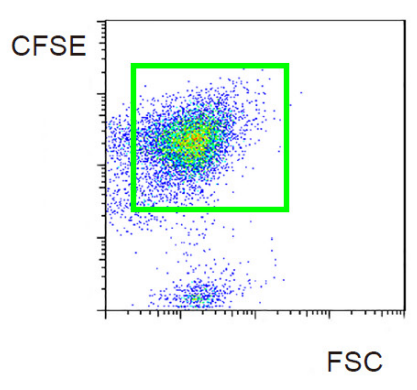

Fig. 1. Isolation, culturing, differentiation and labeling of primary mouse MSCs. Primary mouse MSCs were isolated from male C57BL/6 mice and expanded in culture. (A) After 10 passages' selection of attached cells, the cells were sorted for Stro-1 (red rectangle) by flow cytometry to get rid of contaminating cells. (B-D) Differentiation assays were performed, including Alcian blue staining (B), Von kossa staining (C) and Oil red O staining (D). (E-F) Immediately before transplantation, the MSCs were labeled with CFSE (E), and purified (green rectangle) by flow cytometry (F). Scale bars are $50 \mu \mathrm{m}$.

2A) and percentage of LV fractional shortening (LVFS) (Fig. 2B) in mice transplanted with MSCs compared to PBS control. Masson's Trichrome-stained sections showed markedly reduced levels of LV fibrosis, by representative images (Fig. 2C), and by quantification (Fig. 2D). Moreover, cardiac vessel density was quantified by CD31 positivity, showing significant increase in the LV from the mice that received MSCs after AMI (Fig. 2E-F). These data suggest that grafted MSCs may improve heart function after cardiac muscle injury in isogenic mice.

Direct contribution of grafted MSCs to cardiac muscle cells was very limited

We next examined whether grafted MSCs may contribute to the regeneration of the injured cardiac muscle by direct differentiation. Although we detected many CFSE-positive MSCs in the injured heart of MSCs-treated mice, it is technically difficult to evaluate and quantify the CFSE-positive cardiac muscle cells (Fig. 3A). Therefore, we digested the mouse 

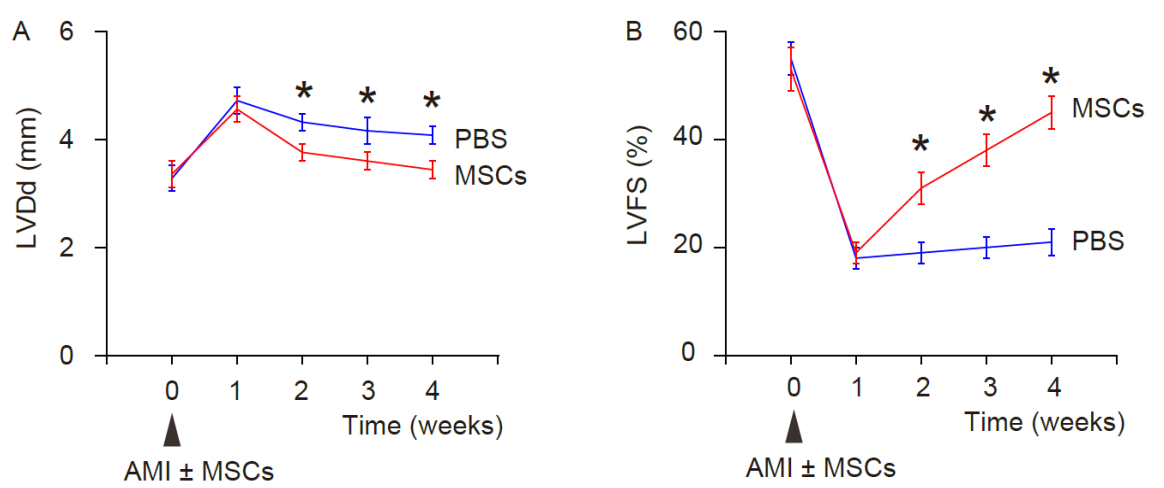

C

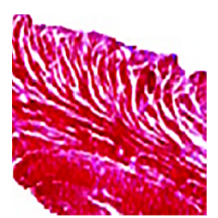

PBS

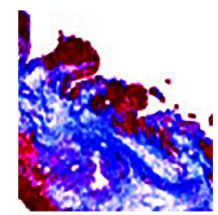

$\mathrm{AMI}+\mathrm{PBS}$

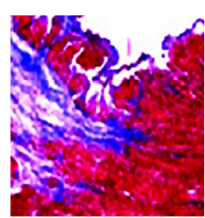

$\mathrm{AMI}+\mathrm{MSCs}$
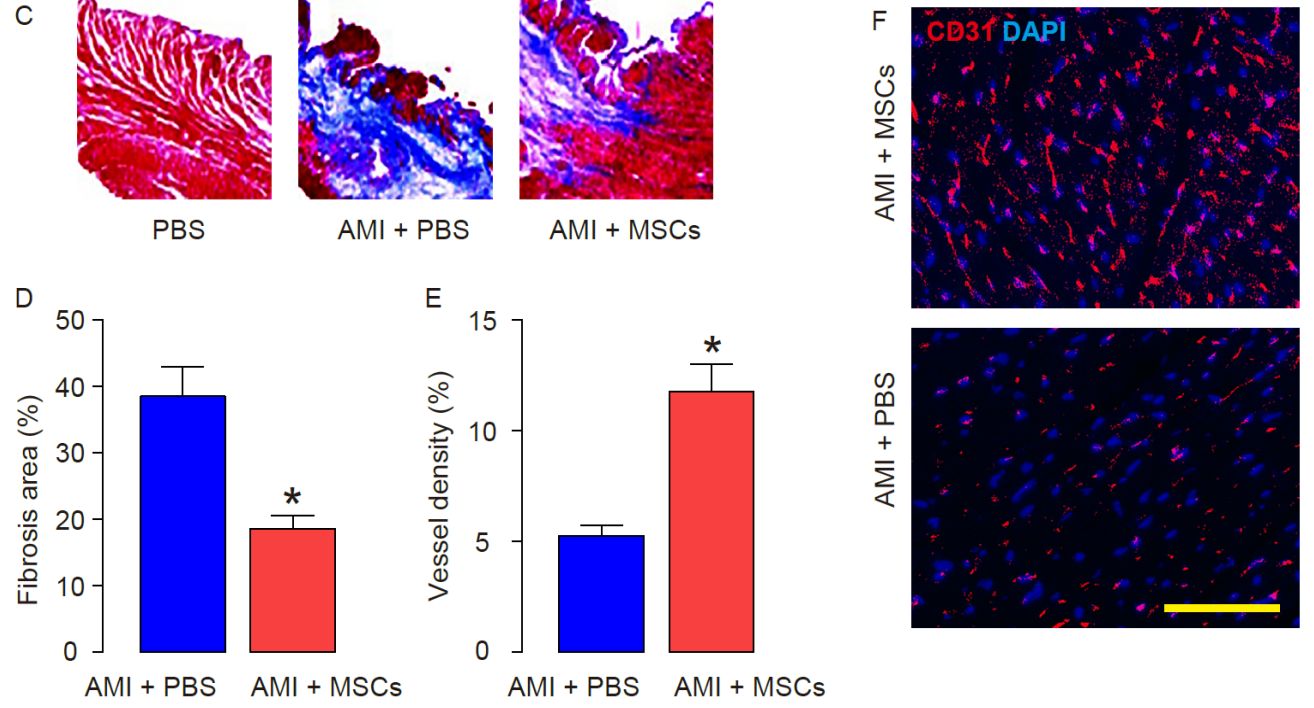

Fig. 2. Grafted MSCs improved heart function after cardiac muscle injury. CFSE-labeled MSCs were transplanted immediately after induction of AMI. (A-B) LV end-diastolic and end-systolic dimensions were measured and functional shortening was determined before AMI (week 0) and on 1, 2, 3 and 4 weeks postAMI. The echocardiography 2, 3, 4 weeks post-AMI showed significantly improved LV diastolic dimensions (LVDd) (A) and percentage of LV fractional shortening (LVFS) (B) in mice transplanted with MSCs compared to PBS control. (C-D) Masson's Trichrome-stained sections showed markedly reduced levels of LV fibrosis, by representative images (C), and by quantification (D). (E-F) Cardiac vessel density was quantified by CD31 positivity, shown by quantification (E), and by representative images (in red) (F). DAPI is nucleus staining (in blue). Scale bars are $50 \mu \mathrm{m} . *^{*}$ p $<0.05$.

heart and labeled the digests with M-cad, a specific cardiac muscle cell marker in the mouse heart. Flow cytometry was applied to objectively analyze the contribution of CFSE-labeled MSCs to cardiac muscle cells. Our data show that fewer than $0.1 \%$ CFSE-positive cells were M-cad-positive, and in all M-cad-positive cells, the percentage of CFSE-positive cells was less than $0.001 \%$ (Fig. 3B). These data suggest that a direct contribution of MSCs to cardiac muscle cells may be very limited. Moreover, the heart digests were also incubated with an endothelial cell marker, CD31, to evaluate the contribution of CFSE-positive MSCs to endothelium by flow cytometry. Our data show that less than $0.1 \%$ CFSE-positive cells were CD31-positive, and in all CD31-positive cells, the percentage of CFSE-positive cells was less than $0.01 \%$ (Fig. 3C). These data suggest that a direct contribution of MSCs to endothelium is also limited. 
Fig. 3. Grafted MSCs did not differentiate into cardiac muscle cells. (A) Representative immunostaining image in MSCs-grafted AMI-heart. M-cad: M-cadherin. DAPI: nucleus staining. (B) Flow cytometry was applied to M-cad-stained heart digests from mice that received MSCs and AMI. Less than $0.1 \%$ CFSE-positive cells were M-cad-positive, and in all M-cad-positive cells, the percentage of CFSE-positive cells was less than $0.001 \%$, suggesting a very limited direct contribution of MSCs to regenerated cardiac muscle cells. (C) The heart digests were also incubated with an endothelial cell marker, CD31,

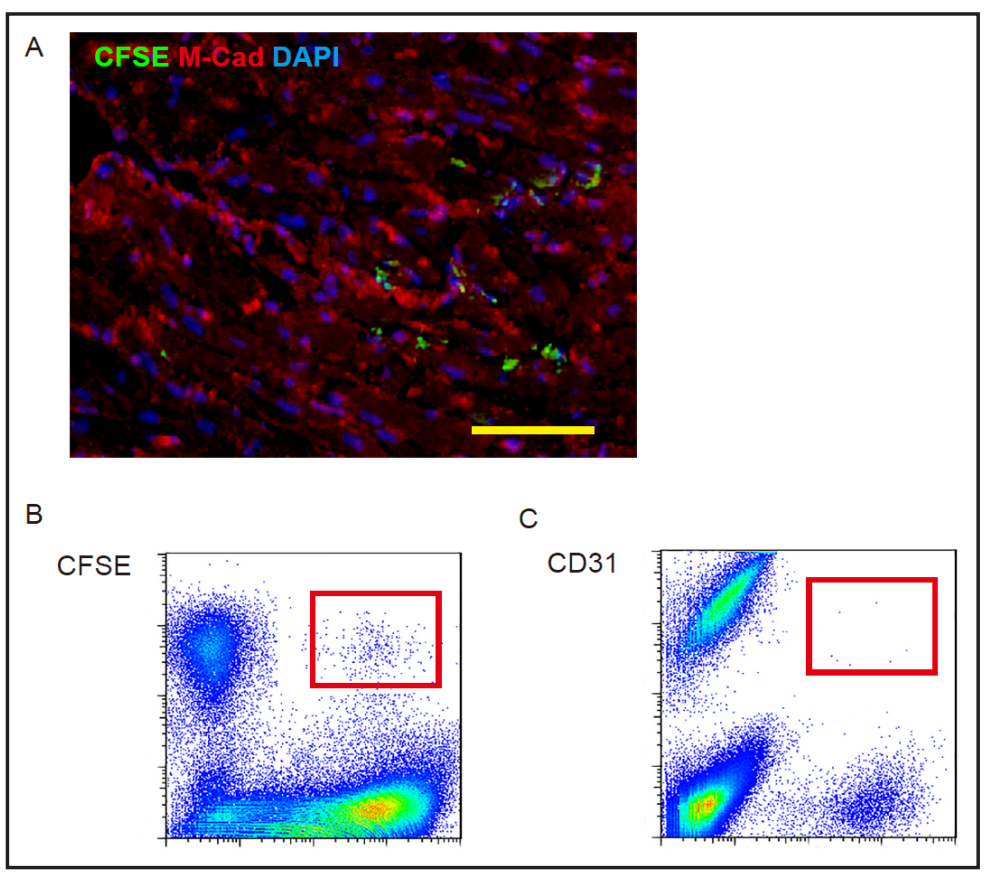

to evaluate the contribution of CFSE-positive MSCs to endothelium by flow cytometry. Our data show that fewer than 0.1\% CFSE-positive cells were CD31-positive, and in all CD31-positive cells, the percentage of CFSE-positive cells was less than $0.01 \%$. These data suggest that a direct contribution of MSCs to endothelium is also limited. Scale bar is $50 \mu \mathrm{m}$.

MSCs produced PLGF to promote neovascularization after cardiac muscle injury

Since transplantation of MSCs significantly increased heart vessel density after cardiac muscle injury, we hypothesized that MSCs may produce angiogenic factors to promote neovascularization. We examined levels of VEGF family members in MSCs, and found that MSCs expressed high levels of VEGF-A and PLGF, and low or null levels of other members. In order to figure out the roles of VEGF-A and PLGF in MSCs in cardiac regeneration, we knocked down VEGF-A or PLGF in MSCs by transduction with lenti-viruses expressing shVEGF-A or shPLGF, respectively, which was assured by examining VEGF-A and PLGF levels by RT-qPCR (Fig. 4A), and by ELISA on cell extracts and conditioned media (Fig. 4B). MSCs that were transduced with null virus were used as a control. Of note, knockdown of VEGF-A in MSCs did not affect PLGF levels, and vice versa (Fig. 4A-B).

We then transplanted MSCs-shVEGF-A, MSCs-shPLGF, and control cells into AMI-mice, respectively. LV functions were measured before AMI (week 0 ) and on 1, 2, 3 and 4 weeks post-AMI. The echocardiography 2, 3, 4 weeks post-AMI showed significant impairment of the improved LV diastolic dimensions (LVDd) (Fig. 4C) and percentage of LV fractional shortening (LVFS) (Fig. 4D) by MSCs transplantation, when PLGF, but not VEGF-A, was inhibited in MSCs. Masson's Trichrome-staining also showed marked impairment of reduced levels of LV fibrosis, when PLGF, but not VEGF-A, was inhibited in MSCs (Fig. 4E). Moreover, the enhanced neovascularization by MSCs appeared to be abolished by PLGF knockdown in MSCs (Fig. 4F). These data suggest that grafted MSCs improve heart function after cardiac muscle injury through PLGF-mediated neovascularization.

PLGF may function through ERK/MAPK and PI3k/Akt signaling pathways in endothelial cells and macrophages after cardiac muscle injury

Since Flt-1 (VEGFR1) is the unique binding receptor for PLGF, we thus hypothesize that the target cells in this model for PLGF may be two Flt-1-expressing cell types, endothelia cells and macrophages [11, 24-26]. To find out the involved downstream signal cascades of 


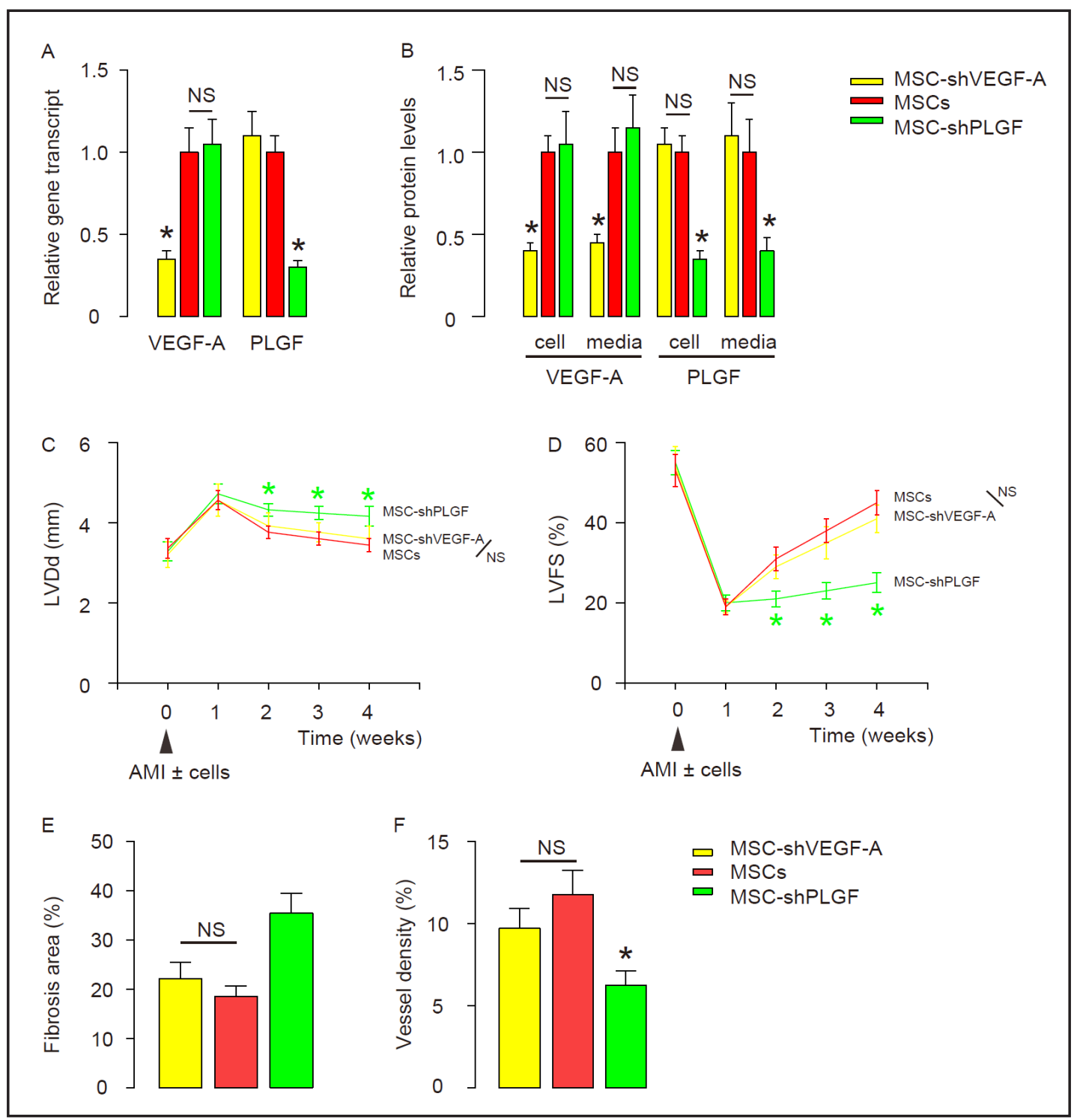

Fig. 4. MSCs produced PLGF to promote neovascularization after cardiac muscle injury. (A-B) VEGF-A or PLGF was knocked down in MSCs by lenti-viruses expressing shVEGF-A or shPLGF, respectively, which was assured by examining VEGF-A and PLGF levels by RT-qPCR (A), and by ELISA on cell extracts and conditioned media (B). MSCs that were transduced with null virus were used as control (MSCs). (C-F) MSCs-shVEGF-A, MSCs-shPLGF, and control MSCs cells were transplanted into AMI-mice, respectively. (C-D) LVDd (C) and LVFS (D) were measured for LV functions before AMI (week 0) and on 1, 2, 3 and 4 weeks post-AMI. (E) Quantification of Masson's Trichrome-staining for LV fibrosis (F) Quantification of vessel density. *: p<0.05. NS: non-significant.

Flt-1 activation in these cells, we isolated endothelial cells and macrophages from AMI-heart digests by flow cytometry, based on expression of CD31, and F4/80, respectively. These cells were put in culture, treated with/without 10nmol/l recombinant PLGF for 2 hours, and then isolated for protein analyses by Western blot. We found that PLGF significantly induced phosphorylation of Flt-1 in both cell types, and the phosphorylation of ERK1/2 and Akt, two key components for ERK/MAPK and PI3k/Akt signaling pathway, respectively (Fig. 5). These data suggest that PLGF may function through ERK/MAPK and PI3k/Akt signaling pathways in endothelial cells and macrophages after cardiac muscle injury. 
Fig. 5. PLGF may function through ERK/MAPK and PI3k/Akt signaling pathways in endothelial cells and macrophages after cardiac muscle injury. Endothelial cells and macrophages were isolated from AMI-heart digests by flow cytometry, based on expression of CD31, and F4/80, respectively. These cells were put in culture, treated with/ without recombinant PLGF for 2 hours, and then isolated for protein analyses by Western blot. pFlt1: phosphorylated Flt-1. pERK1/2: phosphorylated ERK1/2. pAkt: phosphorylated Akt.

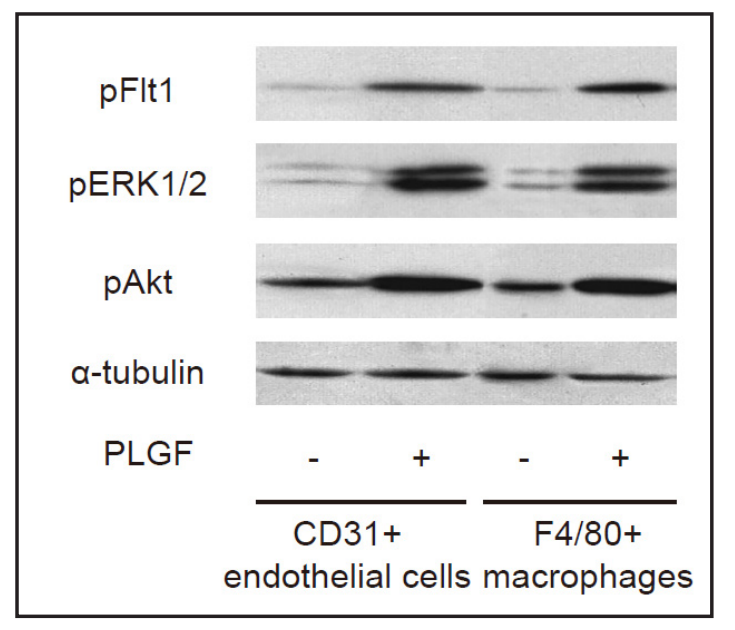

\section{Conclusion}

Production and secretion of PLGF, but not of VEGF-A in MSCs, were essential for the MSCs-induced neovasularization after cardiac muscle injury to facilitate cardiac muscle repair.

\section{Discussion}

Transplantation of bone-marrow derived stem/progenitor cells has an established therapeutic effect on cardiac muscle repair after injury. However, previous studies have controversial results on the underlying mechanism [3-6], which prompted us to address this question by using MSCs, a unique population in bone-marrow derived stem/progenitor cells.

We chose C57BL/6 mice for our study, since it is a strict inbred strain, which allows isogenic transplantation. We first isolated and cultured primary mouse MSCs from male C57BL/6 mice, and assured the phenotypes of MSCs by three differentiation assays. Immediately before transplantation, the MSCs were labeled with CFSE, for the purpose of in vivo lineage tracing. According to previous publications, CFSE (carboxyfluorescein diacetate, succinimidyl ester) is a cell-tagged dye that passively enters the cells, and then is retained within the cells without leaking out. It will dilute during cell proliferation, but the high level of fluorescence allows the daughter cells to be detected within 5-7 cell cycles [16, 27]. Thus, this method is proper for our study.

In order to evaluate the effect of transplantation of MSCs on injured heart, we transplanted CFSE-labeled MSCs immediately after induction of AMI. Significant improvement in LVDd and LVFS, reduced fibrosis, and increased vascularization in mice transplanted with MSCs demonstrate a role for MSCs in cardiac muscle repair. CFSE-labeling of the grafted MSCs allows lineage tracing of these cells. By objective analysis of heart digests by flow cytometry, we found very limited contribution, suggesting that a direct contribution of MSCs to regenerated cardiac muscle cells is very limited, consistent with the results from majority of previous studies [28-30].

Since neovascularization is critical for tissue repair, we thus examined the angiogenic factors in MSCs for promoting neovascularization. We examined levels of VEGF family members in MSCs, and found that MSCs expressed high levels of VEGF-A and PLGF, but low or null levels of other members. In order to figure out the roles of VEGF-A and PLGF in MSCs in cardiac regeneration, we knocked down VEGF-A or PLGF in MSCs by lenti-viruses expressing shVEGF-A or shPLGF, respectively. Of note, it is important to show that knockdown of VEGF-A in MSCs did not affect PLGF levels, and vice versa, which allows us to analyze the effect of VEGF-A and PLGF in MSCs separately. We then transplanted MSCs-shVEGF-A, MSCs-shPLGF, and control cells into AMI-mice, respectively. Our data show that the beneficial effects of 
MSCs on LV functions, reduction in fibrosis and augmented neovascularization were all impaired by knocking down PLGF, but not VEGF-A, in MSCs. These data are consistent with an essential role of PLGF in pathological vascularization [31], which highlights PLGF as a key factor during cardiac muscle repair.

Although it may be attractive to provide exogenous PLGF to the AMI-mice that received shPLGF-MSCs to see if it may rescue the therapeutic effect, technical difficulties prevent its realization. First, systemic supply of PLGF via circulation or via i.p. injection exerts significant off-target effect, since Flt-1 is expressed in all the tissues and organs. Second, exogenous PLGF provision in a subcutaneous implanted mini-osmotic pump suffers from similar shortcoming, and was found to fail to provide a comparable PLGF levels to compensate for the loss of PLGF in shPLGF-MSCs. Third, it is not possible to put this implant pump into the chest to release PLGF locally, which appears to be an ideal way of interference [11].

Since Flt-1 (VEGFR1) is the unique binding receptor for PLGF, we thus think that downstream signal cascades of Flt-1 activation may be responsible for transduction of MSC-induced angiogenesis. By analyzing the responses of two Flt-1-expressing cell types, endothelial cells and macrophages [11, 24-26] in the injured heart to PLGF stimulation, we found that both ERK/MAPK and PI3k/Akt signaling pathways may play roles in the signal transduction. Future experiments may be designed to further elucidate the molecular regulation of these pathways in this model.

\section{Disclosure Statement}

None disclosed.

\section{Reference}

1 Losordo DW, Dimmeler S: Therapeutic angiogenesis and vasculogenesis for ischemic disease: Part ii: Cellbased therapies. Circulation 2004;109:2692-2697.

$\longrightarrow 2$ Losordo DW, Dimmeler S: Therapeutic angiogenesis and vasculogenesis for ischemic disease. Part i: Angiogenic cytokines. Circulation 2004;109:2487-2491.

- Murry CE, Soonpaa MH, Reinecke H, Nakajima H, Nakajima HO, Rubart M, Pasumarthi KB, Virag JI, Bartelmez SH, Poppa V, Bradford G, Dowell JD, Williams DA, Field LJ: Haematopoietic stem cells do not transdifferentiate into cardiac myocytes in myocardial infarcts. Nature 2004;428:664-668.

4 Balsam LB, Wagers AJ, Christensen JL, Kofidis T, Weissman IL, Robbins RC: Haematopoietic stem cells adopt mature haematopoietic fates in ischaemic myocardium. Nature 2004;428:668-673.

-5 Fukata M, Ishikawa F, Najima Y, Yamauchi T, Saito Y, Takenaka K, Miyawaki K, Shimazu H, Shimoda K, Kanemaru T, Nakamura K, Odashiro K, Nagafuji K, Harada M, Akashi K: Contribution of bone marrowderived hematopoietic stem/progenitor cells to the generation of donor-marker(+) cardiomyocytes in vivo. PLoS One 2013;8:e62506.

-6 Wu Y, Zhao RC, Tredget EE: Concise review: Bone marrow-derived stem/progenitor cells in cutaneous repair and regeneration. Stem Cells 2010;28:905-915.

-7 Monsefi M, Fereydouni B, Rohani L, Talaei T: Mesenchymal stem cells repair germinal cells of seminiferous tubules of sterile rats. Iranian journal of reproductive medicine 2013;11:537-544.

-8 Zhao QS, Xia N, Zhao N, Li M, Bi CL, Zhu Q, Qiao GF, Cheng ZF: Localization of human mesenchymal stem cells from umbilical cord blood and their role in repair of diabetic foot ulcers in rats. Int J Biol Sci 2013;10:80-89.

9 Fawzy SA, El-Din Abo-Elnou RK, Abd-El-Maksoud El-Deeb DF, Yousry Abd-Elkader MM: The possible role of mesenchymal stem cells therapy in the repair of experimentally induced colitis in male albino rats. International journal of stem cells 2013;6:92-103.

10 Ye L, Zhang P, Duval S, Su L, Xiong Q, Zhang J: Thymosin beta4 increases the potency of transplanted mesenchymal stem cells for myocardial repair. Circulation 2013;128:S32-41. 
11 Cao X, Han ZB, Zhao H, Liu Q: Transplantation of mesenchymal stem cells recruits trophic macrophages to induce pancreatic beta cell regeneration in diabetic mice. Int J Biochem Cell Biol 2014;53:372-379.

12 Song X, Xie S, Lu K, Wang C: Mesenchymal stem cells alleviate experimental asthma by inducing polarization of alveolar macrophages. Inflammation DOI: 10.1007/s10753-014-9954-6.

13 Kusano KF, Pola R, Murayama T, Curry C, Kawamoto A, Iwakura A, Shintani S, Ii M, Asai J, Tkebuchava T, Thorne T, Takenaka H, Aikawa R, Goukassian D, von Samson P, Hamada H, Yoon YS, Silver M, Eaton E, Ma H, Heyd L, Kearney M, Munger W, Porter JA, Kishore R, Losordo DW: Sonic hedgehog myocardial gene therapy: Tissue repair through transient reconstitution of embryonic signaling. Nat Med 2005;11:1197-1204.

14 Tropel P, Noel D, Platet N, Legrand P, Benabid AL, Berger F: Isolation and characterisation of mesenchymal stem cells from adult mouse bone marrow. Exp Cell Res 2004;295:395-406.

15 Zhou B, Cao XC, Fang ZH, Zheng CL, Han ZB, Ren H, Poon MC, Han ZC: Prevention of diabetic microangiopathy by prophylactic transplant of mobilized peripheral blood mononuclear cells. Acta Pharmacol Sin 2007;28:89-97.

16 Xiao X, Guo P, Shiota C, Prasadan K, El-Gohary Y, Wiersch J, Gaffar I, Gittes GK: Neurogenin3 activation is not sufficient to direct duct-to-beta cell transdifferentiation in the adult pancreas. J Biol Chem 2013;288:25297-25308.

17 Urbani S, Caporale R, Lombardini L, Bosi A, Saccardi R: Use of cfda-se for evaluating the in vitro proliferation pattern of human mesenchymal stem cells. Cytotherapy 2006;8:243-253.

18 Xiao X, Prasadan K, Guo P, El-Gohary Y, Fischbach S, Wiersch J, Gaffar I, Shiota C, Gittes GK: Pancreatic duct cells as a source of vegf in mice. Diabetologia 2014;57:991-1000.

19 Li D, Wu J, Bai Y, Zhao X, Liu L: Isolation and culture of adult mouse cardiomyocytes for cell signaling and in vitro cardiac hypertrophy. J Vis Exp DOI: 10.3791/51357.

20 Shen B, Liu X, Fan Y, Qiu J: Macrophages regulate renal fibrosis through modulating tgfbeta superfamily signaling. Inflammation 2014

-21 Wolf CM, Moskowitz IP, Arno S, Branco DM, Semsarian C, Bernstein SA, Peterson M, Maida M, Morley GE, Fishman G, Berul CI, Seidman CE, Seidman JG: Somatic events modify hypertrophic cardiomyopathy pathology and link hypertrophy to arrhythmia. Proc Natl Acad Sci U S A 2005;102:18123-18128.

22 Ning H, Lin G, Lue TF, Lin CS: Mesenchymal stem cell marker stro-1 is a 75 kd endothelial antigen. Biochem Biophys Res Commun 2011;413:353-357.

23 Lin G, Liu G, Banie L, Wang G, Ning H, Lue TF, Lin CS: Tissue distribution of mesenchymal stem cell marker stro-1. Stem Cells Dev 2011;20:1747-1752.

24 Sica A, Mantovani A: Macrophage plasticity and polarization: In vivo veritas. J Clin Invest 2012;122:787795.

25 Lawrence T, Natoli G: Transcriptional regulation of macrophage polarization: Enabling diversity with identity. Nat Rev Immunol 2011;11:750-761.

26 Xiao X, Gaffar I, Guo P, Wiersch J, Fischbach S, Peirish L, Song Z, El-Gohary Y, Prasadan K, Shiota C, Gittes GK: M2 macrophages promote beta-cell proliferation by up-regulation of smad7. Proc Natl Acad Sci U S A 2014;111:E1211-1220.

-27 Bronner-Fraser M: Alterations in neural crest migration by a monoclonal antibody that affects cell adhesion. J Cell Biol 1985;101:610-617.

-28 Hatzistergos KE, Quevedo H, Oskouei BN, Hu Q Feigenbaum GS, Margitich IS, Mazhari R, Boyle AJ, Zambrano JP, Rodriguez JE, Dulce R, Pattany PM, Valdes D, Revilla C, Heldman AW, McNiece I, Hare JM: Bone marrow mesenchymal stem cells stimulate cardiac stem cell proliferation and differentiation. Circ Res 2010;107:913-922.

29 Noiseux N, Gnecchi M, Lopez-Ilasaca M, Zhang L, Solomon SD, Deb A, Dzau VJ, Pratt RE: Mesenchymal stem cells overexpressing akt dramatically repair infarcted myocardium and improve cardiac function despite infrequent cellular fusion or differentiation. Mol Ther 2006;14:840-850.

-30 Sauer H, Hescheler J, Wartenberg M: Cardiac differentiation of mesenchymal stem cells in sex mis-matched transplanted hearts: Self-repair or just a visit? Cardiovasc Res 2002;56:357-358.

-31 Autiero M, Waltenberger J, Communi D, Kranz A, Moons L, Lambrechts D, Kroll J, Plaisance S, De Mol M, Bono F, Kliche S, Fellbrich G, Ballmer-Hofer K, Maglione D, Mayr-Beyrle U, Dewerchin M, Dombrowski S, Stanimirovic D, Van Hummelen P, Dehio C, Hicklin DJ, Persico G, Herbert JM, Shibuya M, Collen D, Conway EM, Carmeliet P: Role of plgf in the intra- and intermolecular cross talk between the vegf receptors flt 1 and flk1. Nat Med 2003;9:936-943. 


\section{Erratum}

In the article by Zhang et al., entitled “ Mesenchymal Stem Cells Promote Cardiac Muscle Repair via Enhanced Neovascularization " [Cell Physiol Biochem 2015;35:1219-1229 (DOI: 10.1159/000373945)], is a printing error in the affiliations and the corresponding authors address.

The corrected authors and their affiliations are stated correctly here.

Jianfeng Zhang ${ }^{\mathrm{a}, \mathrm{b}} \quad{\text { Yicheng } \mathrm{Wu}^{\mathrm{b}} \quad \text { Anqing Chen }^{\mathrm{a}} \quad \text { Qiang Zhao }}^{\mathrm{a}}$

aDepartment of Cardiac Surgery, Ruijin Hospital, School of Medicine, Shanghai Jiao Tong University, Shanghai, China, ${ }^{b}$ Department of Cardiovascular Surgery, Shanghai Chest Hospital, Shanghai Jiao Tong University, Shanghai, China

Corresponding address:

Department of Cardiac Surgery, Ruijin Hospital, School of Medicine, Shanghai Jiao Tong University, 197 Rui Jin Er Road, Shanghai 200025, (China) 